\title{
Adaptación de la residencia de Cirugía General en Colombia a la pandemia del COVID-19: programa de enseñanza quirúrgica virtual
}

\author{
Adaptation of the residence of general surgery in Colombia to \\ the COVID-19 pandemic: virtual surgical education program.
}

Luis Felipe Cabrera ${ }^{1}$, Carlos Luna Jaspe ${ }^{2}$, Mauricio Pedraza Ciro ${ }^{3}$

\begin{abstract}
Médico, Especialista en Cirugía General, Departamento de Cirugía, Fundación Santa Fe de Bogotá. Profesor de cirugía, Universidad El Bosque y Universidad de los Andes. Bogotá, Colombia. Miembro Comité de Comunicaciones Asociación Colombiana de Cirugía 2 Director del Posgrado Cirugía General, Profesor Titular, Universidad El Bosque, Bogotá, D.C., Colombia

3 Residente de Cirugía General, Universidad El Bosque, Bogotá, D.C., Colombia
\end{abstract}

\section{Resumen}

Esta pandemia le ha dado un giro a la enseñanza quirúrgica, ya que durante este periodo todos los esfuerzos de los servicios quirúrgicos se concentrarán en proporcionar la mejor atención posible a la población afectada por el COVID-I9, al mismo tiempo que se protege y preserva la fuerza laboral quirúrgica. Sin embargo, la educación quirúrgica no debe ser abandonada y los programas de educación virtual quirúrgica continuada pueden ser una opción viable para mitigar el efecto de la pandemia en la formación de los médicos residentes. Al mostrar y compartir esta experiencia queremos abrir la puerta a desarrollar trabajos futuros para evaluar y comparar de forma objetiva la eficacia de estos nuevos métodos de enseñanza en nuestro país.

Palabras clave: COVID-ı9; infecciones por coronavirus; pandemias; cirugía general; educación médica; programas de postgrado.

\begin{abstract}
This pandemic has changed surgical education, since during this period all the efforts of the surgical services will focus on providing the best possible care to the population affected by COVID-I9, while protecting and preserving the surgical workforce. However, surgical education should not be abandoned, and continuing virtual
\end{abstract}

Fecha de recibido: 13/04/2020 - Fecha de aceptación: 17/04/2020

Correspondencia: Luis Felipe Cabrera-Vargas, MD. Dirección: Calle 157 \# 13 B - 20 Casa 10 Conjunto Almeria. Bogotá DC, Colombia. Celular: 311-4342746

Correo electrónico: luis.felipe.cabrera@hotmail.com

Citar como: Cabrera LF, Luna Jaspe C, Pedraza-Ciro M. Adaptación de la residencia de Cirugía General en Colombia a la pandemia del COVID-19: programa de enseñanza quirúrgica virtual. Rev Colomb Cir. 2020;35:256-63/Especial COVID-19. https://doi.org/10.30944/20117582.632

Este es un artículo de acceso abierto bajo una Licencia Creative Commons - BY-NC-ND https://creativecommons.org/licenses/by-ncnd/4.0/deed.es 
surgical education programs may be a viable option to mitigate the effect of the pandemic on the training of surgical residents. By sharing this experience, the possibility to develop future works to objectively evaluate and compare the effectiveness of these new teaching methods in our country.

Key words: COVID-I9; coronavirus infections; pandemics; general surgery; education, medical; health postgraduate programs.

\section{Introducción}

La educación quirúrgica tiene como objetivo proporcionar a los médicos residentes una base de conocimiento quirúrgico, competencia técnica y juicio clínico para el manejo de los pacientes quirúrgicos. Incluso antes de la actual pandemia mundial, la educación quirúrgica ha tenido que responder con nuevas tecnologías, tratamientos y procedimientos, a los cambios dinámicos en el conocimiento de la enfermedad quirúrgica, la demanda en la supervisión de los médicos residentes y las exigencias por la seguridad del paciente ${ }^{\mathrm{I}}$.

Los cambios intensos y rápidos a nivel global generados por la pandemia del COVID-I9, requieren una respuesta diferente a la habitual, de ritmo lento en los modelos de entrenamiento de los médicos residentes, ya que obliga a muchos alumnos a quedarse en casa y focaliza su atención en el manejo de pacientes médicos en estado crítico ${ }^{\mathrm{I}}$. Por esa razón consideramos pertinente mostrar nuestra experiencia en el desarrollo de un programa de enseñanza virtual quirúrgica en Colombia y realizar una revisión crítica de la literatura.

\section{Métodos}

Presentamos la experiencia que hemos tenido hasta la fecha con nuestro proyecto de enseñanza quirúrgica virtual, un producto del grupo de investigación en cirugía general y subespecialidades (GICS) y del semillero de investigación en cirugía general y subespecialidades (SICS) de la Universidad El Bosque, en Bogotá DC, Colombia, el cual inició con la creación de nuestras redes sociales@Cirbosque en Twitter, Facebook e Instagram, el 22 de febrero de 2019.

De forma semanal se llevan a cabo las siguientes actividades:
I. Publicación diaria sobre una patología quirúrgica específica de la semana

2. Imagen de la semana

3. Reto diario de imágenes, para promover el aprendizaje significativo con estímulos visuales

4. Caso quirúrgico de la semana

5. Caso clínico-quirúrgico diario, con el fin de incentivar la discusión y toma de decisiones quirúrgicas de forma académica, con pares de experiencia mundial

6. Publicación internacional recomendada de la semana, la cual es acerca del tema de la semana

7. Promoción de los artículos académicos publicados por nuestro posgrado de Cirugía general en la Universidad El Bosque

8. Promoción y transmisión en vivo de los denominados Grand Rounds mensuales de nuestro posgrado

9. Artista o pintor por semana

IO. Se resaltan a los cirujanos que han hecho historia

II. Se incentiva el liderazgo en los integrantes de nuestro programa de posgrado de cirugía general, mostrándose cada semana como futuros jóvenes cirujanos, promoviendo sus metas y logros.

I2. No hay limitación para el nivel académico de los participantes: estudiantes, internos, médicos residentes y especialistas pueden ser parte activa del proyecto. 
Cada publicación que realizamos va ligado a una tendencia como la de \#SoMe4Surgery. Además se encuentran referenciados los cirujanos más importantes a nivel académico y de redes sociales en el mundo y a nivel local, al igual que las sociedades científicas quirúrgicas nacionales e internacionales más reconocidas como el American College of Surgeons (ACS), la Society of American Gastrointestinal and Endoscopic Surgeons (SAGES), la Sociedad Panamericana de Trauma (SPT), y la Asociación Colombiana de Cirugía (ACC), entre otras, con el fin de difundir de forma masiva la información. Cada publicación trae información académica con sus referencias bibliográficas en forma de imágenes, videos, audios, tablas, esquemas, acrónimos, mapas conceptuales y, en menor medida, textos para darle un soporte sólido, veraz y llamativo a cada una, con el fin de obtener una asociación visual de la información en nuestros médicos residentes ${ }^{2}$. Las publicaciones con casos e imágenes clínicas en nuestro programa de enseñanza virtual respetan la privacidad de los pacientes y tienen autorización de los pacientes y del Comité de Ética de cada institución.

Todo lo anterior es realizado por un especialista en cirugía general y profesor de nuestro posgrado, permitiendo a los médicos residentes una interacción directa, de forma continua, con un par académico de talla mundial, que puede responder de forma veraz y concisa a sus preguntas en tiempo real, sin importar las distancias o ubicaciones geográficas, con el fin de generar un aprendizaje significativo ${ }^{2}$. Esto nos hizo visibles en el mapa mundial de tendencias como \#SoMe${ }_{4}$ Surgery, creada por el doctor Julio Mayol, generando liderazgo, consejería, educación y difusión de la información de forma global en nuestros médicos residentes. Por último, fuimos invitados a mostrar nuestro proyecto en una ponencia magistral durante el Congreso Nacional de Cirugía de la Asociación Colombiana de Cirugía en la ciudad de Bogotá en el año $2019^{2}$.

Con la llegada de la pandemia de COVID-I9, esta crisis mundial sin precedentes ha obligado a los cirujanos a reconsiderar casi todas las facetas de su práctica clínica diaria, cancelando la ciru- gía electiva, disminuyendo el número de intervenciones quirúrgicas de urgencia y limitando el uso de la cirugía mínimamente invasiva. Si bien los efectos finales del COVID-I9 aún están por verse en Colombia, no podemos ignorar el impacto de este nuevo y evolutivo estado de "pandemia” en el entrenamiento quirúrgico. Por lo cual el primero de marzo del 2020, inició la segunda fase de nuestro programa de enseñanza quirúrgica virtual, con el desarrollo de las siguientes actividades diarias:

I. Uso de elementos de protección personal (EPP) obligatorios para todos los médicos residentes en rotaciones clínico-quirúrgicas con riesgo de exposición a pacientes $\mathrm{CO}$ VID-I9 positivos.

2. Mantenimiento del distanciamiento social, con una distancia mínima de un metro

3. Uso obligatorio de tapabocas convencional

4. Médicos residentes de último año como segunda línea de atención, para apoyar a los instructores clínicos en caso de incapacidad por COVID-I9 o colapso de los servicios de salud de nuestra institución base.

5. Uso de la plataforma Meet de Google para comunicación en vivo y reuniones virtuales, la cual es gratuita, no tiene un tiempo límite para cada reunión, es de fácil acceso, no requiere una aplicación específica y es amigable para compartir el contenido de ayuda visual, como presentaciones o exposiciones, videos e imágenes clínicas. Así, de forma diaria se realizaron las siguientes actividades de aprendizaje virtual:

- Revisión académica de tema por un médico residente designado

- Discusión crítica de casos clínicos de @ Cirbosque

- Reto de imágenes de radiología en cirugía de@Cirbosque

- Video aprendizaje de cirugías abiertas y laparoscópicas de@Cirbosque 
- Discusión virtual de artículos científicos

- Revisión de historia de la cirugía

- Charla cultural y artística

- Aprendizaje virtual de nudos quirúrgicos con simuladores hechos en casa, diseñados por los profesores Luis Felipe Cabrera (@PipeCabreraV) y Stiven Aparicio, link: https://www.youtube.com/watch?v=flPUEISDnyY

- Enseñanza de forma virtual sobre suturas: tipo Lembert, Cushing, Connell Mayo, Gambee, Carrell y Paracaídas.

- Aprendizaje virtual de laparoscopia con simuladores hechos en casa, diseñados por el profesor Jean Pulido (@JAPS_MD), link: https://www.youtube.com/watch?v=kUXD6J_6eag

- Revista clínica virtual

- Evaluación objetiva de lo aprendido al final de cada semana con exámenes online.

\section{Resultados}

El 22 de febrero de 2019 se creó nuestro programa de educación virtual en redes sociales, @ Cirbosque, con el fin de generar un aprendizaje significativo en nuestros médicos residentes y seguidores, y en I4 meses hemos realizado hasta la fecha I0.587 publicaciones académicas, con un crecimiento del 77,I \% mensual. Alcanzamos 5.396 seguidores, con un crecimiento promedio mensual de 426 seguidores, II.24I.00o impresiones, con un crecimiento mensual del $56 \%$, I2I.IO 5 mil visitas, con un crecimiento mensual del $28,9 \%$ y I3.966 menciones, con un crecimiento mensual del $88,3 \%$.

El primero de marzo de 2020 se inició la segunda fase de nuestro programa de educación virtual, empleando la plataforma Meet de Google, alcanzando 13 revisiones académicas, 5 revisiones de casos clínicos, 3 retos de imágenes de radiología, 2 ejercicios de video aprendizajes, I revista clínica virtual, 2 revisiones de la historia de la cirugía y i charla cultural artística. La asis- tencia mínima a las reuniones virtuales fue de 27 participantes y la máxima de I02 participantes, con un promedio de 47 participantes, incluyendo estudiantes de pregrado, internos, médicos residentes e instructores clínicos. El promedio ponderado de notas sobre un total de 5 puntos fue de 3.9I.

\section{Discusión}

El programa de educación quirúrgica virtual es una respuesta al cambio en la educación a nivel mundial y a la pandemia del COVID-I9, con el fin de mantener la integridad de la capacitación quirúrgica, al tiempo que se protege a nuestros médicos residentes y se asegura la sostenibilidad de una fuerza laboral crítica de segunda línea para nuestro sistema de salud, en caso de necesitarlos ${ }^{\mathrm{I}, 2,3}$.

Es necesario tener en mente que la pandemia del COVID-I9 será un evento único en la vida de nuestros médicos residentes. La rápida generación y disponibilidad de datos y recomendaciones sobre esta nueva enfermedad y su impacto en la cirugía, brinda a los médicos residentes la oportunidad de cultivar habilidades en el procesamiento y la traducción de la evidencia publicada de manera oportuna ${ }^{\mathrm{I}, 4}$.

Estas medidas que permiten cambiar la forma en la que se enseña la cirugía, le dan la posibilidad a los programas de posgrado de mantener de forma prioritaria la salud y la seguridad de los médicos residentes, como un valor fundamental de todos los entes de formación. Deben eliminarse los riesgos de transmisión innecesarios asociados con las actividades educativas en cirugía general y adaptar al máximo posible estas actividades para el aprendizaje remoto. Cada residente debe conocer la importancia de reportar de forma temprana la aparición de síntomas respiratorios o gastrointestinales y quedarse en casa, comunicándose al contacto de epidemiología y control de infecciones de cada hospital para solicitar instrucción sobre el aislamiento y las pruebas COVID-I9 ${ }^{1,5}$.

Se deben hacer esfuerzos para restringir a los médicos residentes en actividades clínico-qui- 
rúrgicas no esenciales y limitar el número diario de personas en entrenamiento en los servicios de urgencias, hospitalización y salas de cirugía. Todos los médicos residentes deben instruirse en mantener el distanciamiento social efectivo, el uso adecuado y completo de los elementos de protección personal (EPP), y el uso y reúso adecuado de las máscaras N95, garantizando su disponibilidad. La salud física y mental de los médicos residentes debe ser monitorizada, aumentando los esfuerzos con el apoyo de bienestar universitario para mantenerla ${ }^{\mathrm{I}, 4,6}$.

Las habilidades de promoción y comunicación responsable de la información clínica y científica se pueden desarrollar mediante el uso de plataformas digitales, como las redes sociales, tal como lo hacemos con @Cirbosque, que no solo ha influido en los pares académicos quirúrgicos, sino también en los líderes del sistema de salud.

Anticipando la reestructuración del flujo de trabajo y la disminución en el volumen de cirugías electivas y de urgencia, se deben desarrollar oportunidades de aprendizaje para los médicos residentes por fuera del hospital, permitiendo el aprendizaje remoto, tal como lo podemos evidenciar en nuestro programa de enseñanza virtual con el uso de casos clínicos, imágenes de radiología y videos quirúrgicos. A través del liderazgo efectivo y la creación de roles en respuesta a la pandemia, los instructores clínicos pueden inspirar y fomentar habilidades efectivas no quirúrgicas, como trabajo en equipo, solución de problemas, gestión de crisis, liderazgo y educación, formando a los médicos residentes como futuros líderes quirúrgicos, como lo vemos en las actividades de promoción del liderazgo de @ Cirbosque ${ }^{1,3,7,8}$.

A pesar de la crisis de la salud pública por la pandemia del COVID-I9, no debe abandonarse un plan de estudios educativo formal para los médicos residentes, tal como lo hemos demostrado con nuestro programa de educación virtual. Los instructores clínicos asintomáticos que estén en cuarentena o que tengan tiempo libre entre sus actividades laborales clínico-quirúrgicas, deben estar disponibles para dirigir y partici- par en la capacitación quirúrgica remota de los médicos residentes, a través de las plataformas establecidas.

La educación virtual permite compartir recursos y actividades entre diferentes programas y niveles académicos (estudiantes, internos y médicos residentes), y brinda oportunidades para desarrollar relaciones educativas ampliadas, más allá de los límites habituales de una sola institución, ciudad o país. Las conferencias educativas y las sesiones didácticas pueden ofrecerse virtualmente, tal como lo hacemos en nuestro programa. Las plataformas virtuales usadas para el aprendizaje remoto deben estar disponibles para todos los alumnos, de fácil acceso y manejo, como la que utilizamos para este propósito en nuestro programa.

Aunque la simulación práctica en habilidades quirúrgicas puede ser difícil, se deben buscar enfoques innovadores para el desarrollo de estas actividades, como la realización de tareas con retroalimentación en video, tal y como lo proponemos en nuestro programa con el entrenamiento en nudos y en laparoscopia, con el uso de simuladores hechos en casa. Se debe seguir desarrollando la investigación y fomentando la productividad académica de los médicos residentes, completando el registro de casos en las bases de datos, enviando publicaciones para evaluación en las diferentes revistas científicas indexadas y realizando las correcciones solicitadas a las publicaciones en curso, tal como lo hacemos en el grupo de investigación en cirugía general y subespecialidades (GICS) y el semillero de investigación en cirugía general y subespecialidades (SICS) de la Universidad El Bosque ${ }^{\text {1,9-II }}$.

Deben desarrollarse estrategias de redistribución para las actividades académicas y prácticas, con el fin de no solo enseñar cirugía general, sino de involucrar y preparar a los médicos residentes para nuevos roles no quirúrgicos como respuesta a la pandemia, en caso de tener un aumento desmedido de pacientes COVID-I9. Estas actividades de preparación no quirúrgicas las hemos desarrollado con el apoyo del postgrado de anestesiología y reanimación, para la capacitación de 
nuestro personal en intubación segura y ventilación mecánica básica en pacientes COVID-I9 positivos ${ }^{1,3,12,13}$.

Las razones por las cuales el arte y la historia deberían tener un papel importante en la educación médica y quirúrgica de un médico residente están definidas en la necesidad biológica de autoexpresión del hombre, lo cual se demuestra con los dibujos rupestres del hombre prehistórico, como prueba de la necesidad del hombre de expresar sus emociones. La autoexpresión puede tomar muchas formas, desde el jardinero que adorna y poda un atractivo jardín hasta el cirujano que realiza un procedimiento quirúrgico. Existe prueba de que en todos los humanos está presente el deseo de alguna actividad creativa y que esto da satisfacción al creador, tal como al cirujano que completa una gastrectomía total. Los cirujanos también poseen esta necesidad, y muchos esfuerzos artísticos son comprendidos por estos en cada procedimiento quirúrgico, porque permiten la autoexpresión.

Estudiar arte en cirugía ayuda a los médicos residentes a ver con gran detalle; en cirugía, desarrollar una observación precisa conduce al razonamiento y la deducción lógica, y así como a tomar decisiones acertadas. Los cirujanos necesitan obtener y mejorar habilidades tales como la observación, la percepción precisa, el conocimiento de los detalles y la capacidad de juzgar rápidamente, para poder resolver de forma efectiva problemas en cirugía. Estas cualidades reemplazan las impresiones con hechos, y la profesión quirúrgica se ocupa de los hechos. El cirujano con un ojo entrenado notará más rápidamente una anatomía anormal en la vía biliar de su paciente.

Es probable que el cirujano que estudió algo de arte y aprendió a observar con cuidado y precisión, haya aumentado sus habilidades de diagnóstico intraoperatorio, pues alcanza una percepción aguda de la anatomía intraoperatoria, constituyéndose en una herramienta valiosa que nos ayuda a descubrir nuevas dimensiones, formas o planos y diferencias sutiles de color, cambiando la interpretación de lo que vemos.
Además, ayuda a compensar la exposición a largas horas de trabajo, tensión frecuente, ansiedad, estrés y traumas emocionales ocasionales de nuestra profesión quirúrgica. Por todo lo anterior es que en nuestro programa virtual llevamos a cabo sesiones de arte e historia de la cirugía, como parte de la formación de nuestros residentes ${ }^{14-16}$.

La certificación quirúrgica de los médicos residentes por año y la graduación de los de último año, se debe modificar y flexibilizar, a medida que toda la comunidad médica se moviliza en torno a la respuesta de la pandemia del COVID-19, ya que el impacto desmoralizador del retraso indefinido para poder completar la formación quirúrgica en ellos puede ser devastador. Es posible hacer ajustes a los programas para permitir que alumnos competentes se gradúen y se unan a la fuerza laboral, tal como lo han llevado a cabo la American Board of Surgery y el Royal College of Surgeons of England, liderando el camino al anunciar exenciones por dificultades para los mínimos de registro de casos y planes para reprogramar los exámenes de certificación. Sin embargo, se deben mantener los estándares rigurosos para la competencia quirúrgica, mientras se adaptan las expectativas de los médicos residentes durante la pandemia. Los directores de programa deberán evaluar y considerar la competencia del médico residente de forma individual, para determinar si está preparado para ingresar a la práctica no supervisada de la cirugía ${ }^{1,3,17}$.

Por último, todos los programas deben preparar a sus médicos residentes para una era "pos COVID-I9", ya que llegará el momento en que se deba retomar el ritmo normal de los servicios quirúrgicos. Todas las instituciones van a enfrentar retrasos en casos clínicos y quirúrgicos y tendremos un aumento considerable en las experiencias prácticas quirúrgicas disponibles para los residentes en el día a día, por lo cual los programas deben ser flexibles para que los residentes puedan asistir a estos procedimientos adicionales y cumplir con el desarrollo de las capacidades técnicas esenciales ${ }^{1,3,17}$. 


\section{Conclusión}

Esta pandemia le ha dado un giro a la enseñanza quirúrgica, ya que durante este periodo todos los esfuerzos de los servicios quirúrgicos se concentrarán en proporcionar la mejor atención posible a la población afectada por el COVID-I9, al mismo tiempo que se protege y preserva la fuerza laboral quirúrgica. Sin embargo, la educación quirúrgica no debe ser abandonada y los programas de educación virtual quirúrgica continuada son una opción viable para mitigar el efecto de la pandemia en la formación de los médicos residentes. $\mathrm{Al}$ compartir esta experiencia propia queremos abrir la puerta para desarrollar trabajos futuros, donde se evalúe y compare de forma objetiva la eficacia de estos nuevos métodos de enseñanza en nuestro medio, usando métodos como encuestas, exámenes y evaluaciones prácticas de habilidades quirúrgicas adquiridas.

\section{Cumplimiento de normas éticas}

Consentimiento informado: Esta publicación es una revisión de la literatura y presenta la experiencia del grupo de investigación en cirugía general y subespecialidades (GICS) y del semillero de investigación en cirugía general y subespecialidades (SICS) de la Universidad El Bosque, en Bogotá DC, Colombia, sin poner en riesgo a los pacientes, por lo que no hay necesidad de un consentimiento informado.

Declaración de conflicto de intereses: Los autores no declararon ningún conflicto de interés.

Fuentes de financiación: Recursos propios de los autores.

\section{Referencias}

I. Daodu O, Panda N, Lopushinsky S, Varghese TK, Brindle M. COVID-I9 - Considerations and implications for surgical learners. Ann Surg. 2020 March 24, [Online ahead of print.]. Disponible en: https://journals.lww. com/annalsofsurgery/Documents/COVID-I9\%20 \%E2\%80\%93\%20Considerations\%2Oand\%2oImplications\%2ofor\%2oSurgical\%2oLearners.pdf

2. Cabrera Vargas LF, Herrera G, Mendoza Zuchini A, Pedraza M, Sánchez S, Pulido J. Aplicación de las redes sociales en la educación del cirujano general. @Cirbosque rompiendo el paradigma. Cir Andal. 2020;31:15-20. https://doi.org/IO.3735I/20203II.2
3. Nassar AH, Zern NK, McIntyre LK, Lynge D, Smith CA, Petersen RP, et al. Emergency restructuring of a general surgery residency program during the coronavirus disease 2019 pandemic. The University of Washington Experience. JAMA Surg. 2020 April 6, [Online ahead of print.]. https://doi.org/IO.IOoI/jamasurg.2020.I219

4. Royal College of Physicians and Surgeons of Canada. Update on Coronavirus Impact to the Royal College. Last Update, March 24, 2020. Disponible en: http:// www.royalcollege.ca/rcsite/documents/about/update-coronavirus-e\#s2. Accessed March I8, 2020.

5. Royal Australasian College of Surgeons. Coronavirus information hub. Disponible en: https://www.surgeons.org/media-centre/coronavirus-information-hub\#Exams. Accessed March 20, 2020.

6. Accreditation Council for Graduate Medical Education. ACGME Response to Coronavirus (COVIDi9). Last Updated, March I8, 2020. Disponible en: https://acgme.org/Newsroom/NewsroomDetails/ArticleID/roIII/ ACGME-Response-to-the-Coronavirus-COVID-I9 . Accessed March 20, 2020

7. National Institutes of Health. Coronavirus disease 19 (COVID-I9). Published 2020. Disponible en: https:/ www.nih.gov/health-information/coronavirus Accessed March 2I, 2020.

8. Worldometer. COVID-I9 coronavirus pandemic. Published 2020. Disponible en: https://www.worldometers. info/coronavirus/ Accessed March 2I, 2020.

9. Centers for Disease Control and Prevention. Interim guidance for implementing home care of people not requiring hospitalization for coronavirus disease 2019 (COVID-19). Published 2020. Disponible en: https:// www.cdc.gov/coronavirus/2019-ncov/hcp/guidance-home-care.html Accessed March 19, 2020.

Io. Washington State Department of Health. What to do if you were potentially exposed to someone with confirmed coronavirus disease (COVID-I9). Disponible en: https://www.doh.wa.gov/Portals/I/Documents/I6oo/ coronavirus/COVIDexposed.pdf Accessed March I8, 2020.

II. Royal College of Surgeons. Coronavirus (COVID-I9). Last Updated, March 24, 2020. Disponible en: https:// www.rcseng.ac.uk/coronavirus/. Accessed March 20, 2020

I2. Centers for Disease Control and Prevention. Interim Guidance for Administrators of US K-I2 Schools and Childcare Programs. Disponible en: https://www.cdc.gov/coronavirus/20I9-ncov/community/schools-childcare/guidance-forschools. html?CDC_AA_refVal=https\%3A\%2F\%2 Fwww. cdc.gov\%2Fcoronavirus\%2F2OI9- ncov\%2Fspecific-groups\%2Fguidance-for-schools.html. Accessed March I9, 2020

13. Santella TM, Kaneva P, Petrucci A, Lebedeva E, Feldman LS, Fried GM, Vassiliou MC. Using social 
media for surgical education: Distraction or opportunity?. Sages 2013 Abstract Archive. Disponible en: https://www.sages.org/meetings/annualmeeting/abstracts-archive/using-social-media-for-surgical-education-distraction-oropportunity/. Accessed March I8, 2020.

I4. Morani AD. Art in medical education: Especially plastic surgery. Aesth. Plast. Surg. 1992;16:213-18. https://doi. org/IO.IOO7/BFooI90066
I5. Di Matteo G. History, art, humanity in surgery. G Chir. 2016;37:236-38.

I6. Fernandes JW, Metka S. Art and plastic surgery. Aesthetic Plast Surg. 2016;40:33I-3. https://doi.org/I0.IO07/ soo266-0I6-06I6-y

I7. American Board of Surgery. ABS Statement on training requirements during COVID-I9. Last Updated, March I9, 2020. Disponible en: http://www.absurgery.org/default. jsp?news_covidı9_training. Accessed March I9, 2020.

\section{Nota de la Editora}

Las Directivas de la Asociación Colombiana de Cirugía y los Directores de los Programas de especialización en Cirugía general de todo el país, quienes conforman la División de Educación, después de haber participado en varias reuniones, recomiendan las siguientes adaptaciones educativas.

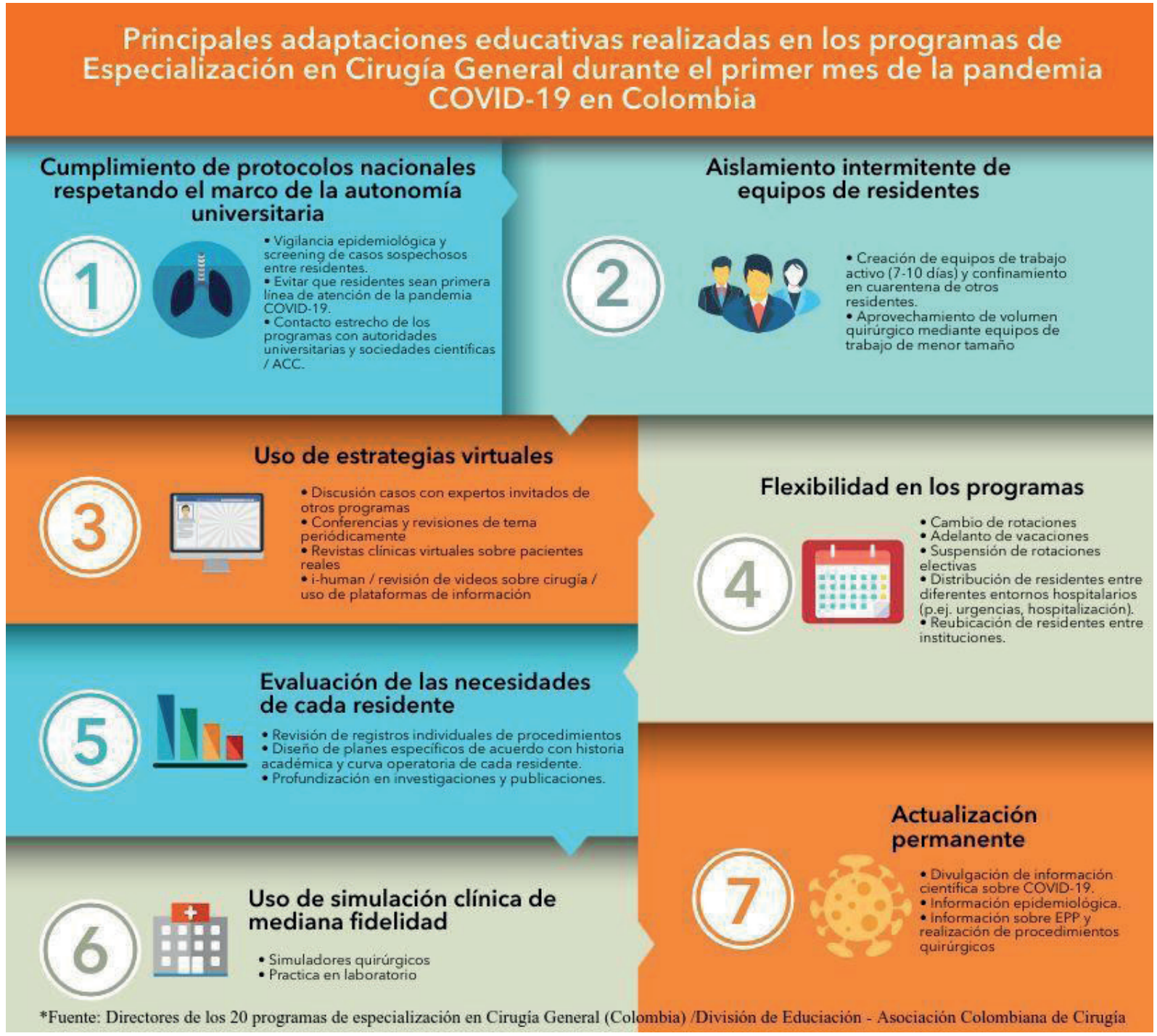

